\title{
HIGHER COMMUTATORS, IDEALS AND CARDINALITY
}

\author{
Charles LaNski
}

\begin{abstract}
For an associative ring $R$, we investigate the relation between the cardinality of the commutator $[R, R]$, or of higher commutators such as $[[R, R],[R, R]]$, the cardinality of the ideal it generates, and the index of the centre of $R$. For example, when $R$ is a semiprime ring, any finite higher commutator generates a finite ideal, and if $R$ is also 2-torsion free then there is a central ideal of $R$ of finite index in $R$. With the same assumption on $R$, any infinite higher commutator $T$ generates an ideal of cardinality at most $2^{\text {card } T}$ and there is a central ideal of $R$ of index at most $2^{\operatorname{card} T}$ in $R$.
\end{abstract}

In this paper we investigate the relative cardinalities of certain subsets of a ring $R$, with the specific goal of generalising work of Hirano [4] who showed the equivalence in a semiprime ring $R$ of the finiteness of the commutator ideal, the additive index of the centre of $R$, and the existence of a central ideal of finite index in $R$. Our aim is to generalise these results to the set of commutators, to higher commutators, and to arbitrary cardinalities, and also to consider the relation between the cardinality of higher commutators and the ideals they generate. A number of papers in the literature relate the cardinality of a ring to that of special subsets. To mention a few of these, in [7], Koh shows that a ring is finite if it has finitely many left zero divisors, and at least two, Hirano extended this to finitely many two-sided zero divisors [3], and recently we considered the relation between the cardinality of a ring and that of the ideal generated by various subsets of nilpotent elements $[8]$. We have also studied the relation between the cardinality of a ring and that of the subring generated by the set of integral elements or the set of symmetric elements in a ring with involution [9].

Throughout the paper, $R$ will denote an associative ring, not necessarily with unit, and $Z(R)=Z$ will be the centre of $R$. For nonempty subsets $A, B \subseteq R$, $L(A, B)=\{[a, b]=$ ab-ba $\mid a \in A$ and $b \in B\}$ is the set of (Lie) commutators of $A$ with $B,[A, B]$ is the additive subgroup of $R$ generated by $L(A, B),\langle A\rangle$ is the subring generated by $A$, and $(A)$ is the ideal of $R$ generated by $A$. We postpone a discussion of higher commutators until later. Our first results will generalise those in [4] by considering $L(R, R)$ rather than the commutator ideal $([R, R])$, and by considering

Received 28 August 1995

Copyright Clearance Centre, Inc. Serial-fee code: 0004-9729/96 \$A2.00+0.00. 
arbitrary cardinalities. Although it is true that $L(R, R)$ finite forces $([R, R])$ to be finite, this not obvious, and it is not immediate that when $L(R, R)$ is infinite, $([R, R])$ has the same cardinality. For future reference, we remind the reader that $[a, b]=-[b, a]$ and that $[, r]: R \rightarrow R$ is an additive map whose kernel is $C(r)$, the centraliser of $r$ in $R$. Out first result is an easy lemma relating the index of $Z$ in $R$ with the cardinality of $[R, R]$. As usual, the index of an additive subgroup $(T,+)$ of $(R,+)$ is denoted by $[R: T]$.

Lemma 1. Let $[R: Z]=\alpha$. If $\alpha$ is finite, then $L(R, R)$ and $[R, R]$ are finite, and if $\alpha$ is infinite, then $\operatorname{card}[R, R] \leqslant \alpha$.

Proof: Let $X=\left\{x_{i}\right\}_{I}$ be a transversal for $(Z,+)$ in $(R,+)$. Clearly card $X=$ card $I=\alpha$ and $R=\bigcup\left\{x_{i}+Z \mid i \in I\right\}$, so $L(R, R)=\left\{\left[x_{i}, x_{j}\right] \mid x_{i}, x_{j} \in X\right\}$ and $L(R, R)$ is finite when $\alpha$ is and otherwise $\operatorname{card} L(R, R) \leqslant \operatorname{card} I \times I=\alpha$. Note that when $L(R, R)$ if finite, then each $[a, b] \in L(R, R)$ is a torsion element since for any integer $n>0, n[a, b]=[a, n b] \in L(R, R)$, forcing $k[a, b]=s[a, b]$ for some $0<k<s$, and so $(s-k)[a, b]=0$. Thus, in this case $[R, R]$ must be finite also. Certainly, if $L(R, R)$ is infinite, then $\operatorname{card}[R, R]=\operatorname{card} L(R, R) \leqslant \alpha$.

Our first example shows that when $R$ is not a semiprime ring, that is when $R$ contains nonzero nilpotent ideals, the conclusion of Lemma 1 that $\operatorname{card}[R, R] \leqslant[R: Z]$ is optimal. In particular, even the ideal $([R, R])$ can be finite regardless of $[R: Z]$.

EXAmple 1. Let $\beta<\alpha$ be cardinals with $\alpha$ infinite, $F$ a field of cardinality $\beta$ with char $F \neq 2$, and $X, Y,\{z\}$ disjoint sets of noncommuting indeterminates over $F$ with $\operatorname{card} X=\operatorname{card} Y=\alpha$. Assume that $I$ is a set with $\operatorname{card} I=\alpha$ and that $X=\left\{x_{i} \mid i \in I\right\}$ and $Y=\left\{y_{j} \mid j \in I\right\}$. In the free algebra $F\{X, Y, z\}$, let $H$ be the ideal generated by $\left\{x_{i} z, z x_{i}, y_{i} z, z y_{i}, z^{2}, x_{i} x_{k}, y_{i} y_{k}, x_{i} y_{j}, y_{j} x_{i}, x_{i} y_{i}-z_{i}, y_{i} x_{i}+z_{i} \mid i, j, k \in I\right.$ and $\left.i \neq j\right\}$ and $R$ the quotient ring $F\{X, Y, z\} / H$. By identifying each indeterminate with its image in $R$, we may write $R=F+F z+\sum_{I} F x_{i}+\sum_{I} F y_{i}$ where all products of elements in $\left\{x_{i}, y_{i}, z\right\}$ are zero except that $x_{i} y_{i}=z$ and $y_{i} x_{i}=-z$ for all $i \in I$. It follows easily that $\operatorname{card} R=\alpha$ and that $Z(R)=F+F z$ is finite or has cardinality $\beta$, so $[R: Z]=\alpha$. Since $\left[x_{i}, y_{i}\right]=2 z \neq 0$, one has $F z=L(R, R)=[R, R]=([R, R])$ has cardinality $\beta$, or is finite.

Our first theorem will be useful in comparing the cardinality of ideals in $Z(R)$ with $[R: Z]$, and will also be used later for results concerning higher commutators.

THEOREM 1 . If $S$ is a nonempty subset of $R$, then both $L(S, R)$ and $([S, R])$ are finite, or else $\operatorname{card} L(S, R)=\operatorname{card}([S, R])$.

Proof: Assume first that $\operatorname{card} L(S, R)=\alpha$ is infinite. Clearly $\operatorname{card}[S, R]=\alpha$ as well, so there is $A=\left\{a_{i}\right\}_{I} \subseteq S$ with $\operatorname{card} A=\operatorname{card} I \leqslant \alpha$ and $[S, R]=\sum_{I}\left[a_{i}, R\right]$. 
For each $i \in I$ define $f_{i}: R \rightarrow[S, R]$ by $f_{i}(r)=\left[a_{i}, r\right]$. Now $f_{i}$ is additive and $f_{i}(R)=\left[a_{i}, R\right] \subseteq[S, R]$ has cardinality at most $\alpha$, so $\left[R\right.$ : $\left.\operatorname{Ker} f_{i}\right] \leqslant \alpha$. Note that $\operatorname{Ker} f_{i}=C\left(a_{i}\right)$, the centraliser of $a_{i}$ in $R$ and let $A_{i}=\left\{x_{i j}\right\}_{J(i)}$ be a transversal for $C\left(a_{i}\right)$ in $R$. Clearly, $\operatorname{card} A_{i}=\operatorname{card} J(i) \leqslant \alpha$. The identity $[x, y z]=y[x, z]+[x, y] z$ shows first that $\left[a_{i}, R\right]$ is a $C\left(a_{i}\right)$ bimodule, and then that $R\left[a_{i}, R\right]=\left(\bigcup_{J(i)}\left\{x_{i j}+C\left(a_{i}\right)\right\}\right)\left[a_{i}, R\right] \subseteq \bigcup_{J(i)}\left\{x_{i j}\left[a_{i}, R\right]+\left[a_{i}, R\right]\right\} \subseteq \sum_{J(i)} x_{i j}\left[a_{i}, R\right]+\left[a_{i}, R\right]$. It follows that $[S, R]+R[S, R]=[S, R]+\sum_{i} R\left[a_{i}, R\right]=[S, R]+\sum_{i, j} x_{i j}\left[a_{i}, R\right]$ has cardinality at most $\alpha$. A similar computation using the fact that $\left[a_{i}, R\right]$ is a $C\left(a_{i}\right)$ bimodule shows that $([S, R])=[S, R]+\sum_{i, j} x_{i j}\left[a_{i}, R\right]+\sum_{i, j}\left[a_{i}, R\right] x_{i j}+\sum_{i, j, k} x_{i j}\left[a_{i}, R\right] x_{i k}$, proving the theorem when $\alpha$ is infinite. Finally, when $\alpha$ is finite, then as in Lemma $1, L(S, R)$ consists of torsion elements, so $[S, R]$ is finite. But now, following the argument just given shows that $([S, R])$ is also finite, completing the proof.

Corollary 1.1. When $L(R, R)$ is finite so is $([R, R])$, and otherwise $\operatorname{card} L(R, R)=\operatorname{card}([R, R])$.

Corollary 1.2. If $[R: Z]=\alpha$, then $([R, R])$ if finite when $\alpha$ is, and $\operatorname{card}([R, R]) \leqslant \alpha$ when $\alpha$ is infinite.

Proof: By Lemma 1, either $L(R, R)$ is finite or card $L(R, R) \leqslant \alpha$, so applying Theorem 1 with $S=R$ finishes the proof.

We use Corollary 1.2 to compare the size of ideals in $Z$ with $[R: Z]$. When $[R: Z]$ is finite, $Z$ must contain an ideal of $R$ of finite index in $R$ by [4, Theorem 1$, p.364], or more generally by a result of Lewin [11, Lemma $1, p .85]$ on subrings of finite index. Consider the situation when $R$ is a noncommutative prime ring with $Z \neq 0$. For $a \in R-Z, Z a \subseteq R-Z$ and $\operatorname{card} Z a=\operatorname{card} Z$, so since $Z a$ must be in some transversal of $Z$ in $R$, it follows that $[R: Z] \geqslant \operatorname{card} Z$. Therefore, either $R$ is finite or $\operatorname{card} R=\max \{[R: Z], \operatorname{card} Z\}=[R: Z]$ and the ideal $(0) \subseteq Z$ satisfies $[R:(0)]=[R: Z]$, so when $R$ is an infinite prime ring, there is an ideal in the centre of $R$ whose index is $[R: Z]$. However, it is not always true for infinite rings $R$ that $Z$ contains an ideal of $R$ of index $[R: Z]$. The following theorem describes the general case.

THEOREM 2. If $[R: Z]=\alpha$, then there is an ideal $I$ of $R$ with $I \subseteq Z$ and $[R: I] \leqslant 2^{\alpha}$ when $\alpha$ is infinite, and $[R: I]$ is finite when $[R: Z]$ is finite.

PRoOF: We assume that $\alpha$ is infinite but note that it will be clear from our argument that when $\alpha$ is finite, $[R: I]$ is finite also. If $B=([R, R])$, then by Corollary $1.2 \operatorname{card} B \leqslant \alpha$. Represent $R$ in $\operatorname{End}(B,+)$ by left multiplication; that is, define $F: R \rightarrow \operatorname{End}(B)$ by $F(r)(b)=r b$. Clearly, $F$ is a ring homomorphism, 
and since card End $(B) \leqslant \alpha^{\alpha}=2^{\alpha}$, if $J=\operatorname{Ker} F=l(B)$, the left annihilator of $B$, then $[R: J] \leqslant 2^{\alpha}$. Using $[R: Z]=\alpha$ yields $[R: J \cap Z] \leqslant 2^{\alpha}$, so it suffices to prove that $J \cap Z$ is an ideal of $R$. To show this, let $x \in J \cap Z$ and $r, s \in R$. Then $[x r, s]=x[r, s]+[x, s] r=0$ since $x \in l(([R, R]))$ and $x \in Z$, so $x r \in Z$, finishing the proof.

In Theorem 2, the appearance of $2^{\alpha}$ when $\alpha$ is infinite is certainly different from the situation when $[R: Z]$ is finite, as in [4]. We have observed that when $R$ is a prime ring, there is always an ideal $I$ in $Z$ with $[R: I]=[R: Z]=\alpha$, when $\alpha$ is infinite. To see that our conclusion that $[R: I] \leqslant 2^{\alpha}$ is not merely a consequence of our particular approach, we present an example of a semiprime ring for which the bound of $2^{\alpha}$ is required.

EXAMPLE 2. Let $\alpha$ be an infinite cardinal, $E$ a set with $\operatorname{card} E=\alpha, M_{2}(F)$ the ring of $2 \times 2$ matrices over the countable (or finite) field $F$, and set $A=\prod_{E} M_{2}(F)$, the infinite direct product of $\alpha$ copies of $M_{2}(F)$. Regarding $A=\left\{g: E \rightarrow M_{2}(F)\right\}$, let $D=\{g \in A \mid g(j)=0$ for all but finitely many $j \in E\}$, the direct sum of $\alpha$ copies of $M_{2}(F)$, and $D_{j}=\{g \in A \mid g(i)=0$ if $i \neq j\}$. Note that $D$ is an ideal of $A$, that card $D=\alpha$, and that $Z(A)=\prod_{E} F I_{2}$, so $\operatorname{card} Z(A)=2^{\alpha}$. If $R=D+Z(A)$, then $R$ is a semiprime ring, card $R=2^{\alpha}$, and $Z(R)=Z(A)$, so $[R: Z(R)]=\alpha$. However, $Z$ contains no nonzero ideal of $R$ since if $I$ is any nonzero ideal of $R$ with $g \in I$ and $g(j) \neq 0$, then $D_{j} g D_{j}=D_{j} \subseteq I$, so $I \not \subset Z$. Consequently $(0)$ is the only ideal of $R$ in $Z$ and we have $[R:(0)]=2^{\alpha}$.

We now consider a converse to Corollary 1.2. Example 1 shows that no useful conclusion about $[R: Z]$ can be drawn from $\operatorname{card}[R, R]$, in general. When $R$ is a semiprime ring then $[R: Z]$ is finite exactly when $([R, R])$ is by [4]. Our next theorem obtains a bound on $[R: Z]$ from the size of $L(R, R)$, so even extends $[4]$ in the finite case.

Theorem 3. Let $R$ be a semiprime ring with $\operatorname{card} L(R, R)=\alpha$. There is an ideal $I$ of $R$ satisfying $I \subseteq Z$ and $[R: I] \leqslant 2^{\alpha}$ when $\alpha$ is infinite, and $[R: I]$ is finite when $\alpha$ is finite. Furthermore, $R$ is a subdirect sum of semiprime images $R_{1}$ and $R_{2}, R_{1}$ is commutative, and $\operatorname{card} R_{2} \leqslant 2^{\alpha}$ when $\alpha$ is infinite. When $\alpha$ is finite, $R=R_{1} \oplus R_{2}$ and $R_{2}$ is finite.

Proof: Assume that $\alpha$ is infinite, so if $B=([R, R])$ then card $B=\alpha$ by Corollary 1.1. As in the proof of Theorem 2, $R$ acts on $B$ by left multiplication, and the kernel of this action, $I=l(B)$, satisfies $[R: I] \leqslant 2^{\alpha}$. Now $[I, I] \subseteq B \cap l(B)=0$ because $R$ is semiprime, and it follows easily [2, Corollary, p.7] that $I \subseteq Z$. Observe that the semiprimeness of $R$ implies that $I=l(B)=r(B)=$ ann $B$, and if $J=$ ann $I$, then $I \cap J=0$ and both $I$ and $J$ are semiprime ideals of $R$. Therefore, $R$ embedds in 
$R / J \oplus R / I, \operatorname{card} R / I=[R: I] \leqslant 2^{\alpha}$, and $R / J$ is commutative since $[R, R] \subseteq B \subseteq J$, completing the proof when $\alpha$ is infinite. When $\alpha$ is finite, $B$ is a finite ideal of $R$, again by Corollary 1.1, so a finite semisimple Artinian ring with identity. Thus $B$ is a direct summand of $R$, say $R=H \oplus B$, and since $[R, R] \subseteq B$ and $H \cap B=0$, it follows that $H \subseteq Z$.

COROLlaRY 3.1. If $R$ is a semiprime ring, $\operatorname{card} L(R, R)=\alpha$, and some $x \in$ $([R, R])$ is (left) regular in $R$, then card $R \leqslant 2^{\alpha}$ when $\alpha$ is infinite and $R$ is finite when $\alpha$ is finite.

Proof: As in the proof of Theorem 3, if $B=([R, R])$ then $\operatorname{card} B=\alpha$ or is finite. But $B$ contains a regular element, so $l(B)=0$, and $R$ embeds isomorphically in $\operatorname{End}(B,+)$. Thus card $R \leqslant 2^{\alpha}$ when $\alpha$ is infinite, and $R$ is finite when $\alpha$ is.

As in Theorem 2, the appearance of $2^{\alpha}$ in Theorem 3 is neither accidental nor simply a consequence of our approach. A natural question is whether replacing $I$ with $Z$ in Theorem 3 might allow one to show that $[R: Z] \leqslant \alpha$ ? Our next example shows that even for prime rings a bound of $2^{\operatorname{card}[R, R]}$ for $[R: Z]$ is necessary when $[R, R]$ is infinite.

EXAMPLE 3. Let $H$ be the ring of all row finite, countably infinite by countably infinite matrices over a countable field $F$. Denote by $M_{0}(F)$ the subring of $H$ of all matrices having only finitely many nonzero entries, and observe that $M_{0}(F)$ is a countable ring. If $\left\{e_{i j}\right\}$ are the usual matrix units in $H$, set $B=\left\{\sum_{i} f_{i} e_{2 i-12 i} \in H \mid f_{i} \in F\right\}$. Since there is a bijection between $B$ and all infinite sequences of elements of $F, B$ is uncountable. Also $B^{2}=0$ and $M_{0}(F) B+B M_{0}(F) \subseteq M_{0}(F)$, so $R=M_{0}(F)+B$ is an uncountable ring with $[R, R] \subseteq M_{0}(F)$, so countable. As in [9, Example 2, p.365], it is easy to see that $R$ is a prime ring since $\left\{e_{i j}\right\} \subseteq R$, and that $Z(R)=0$. Therefore, although $[R, R]$ is countable, $[R: Z]$ is uncountable. A similar example with $Z(R) \neq 0$ can be obtained by including in $R$ all the scalar matrices; that is, by taking $R=$ $M_{0}(F)+B+F 1_{H}$.

A final example on commutators shows that unlike the situation in Theorem 3 when $L(R, R)$ is finite, when it is infinite $R$ may not decompose into a direct sum of a ring of cardinality $L(R, R)$ and a commutative ring.

EXAMPLE 4. Let $F\{X\}$ be the free algebra over the countable field $F$ in an infinite set of noncommuting indeterminates $X$ with card $X=\alpha$, and let $Y$ be another infinite set of commuting indeterminates over $F\{X\}$ with $\operatorname{card} Y=\beta$. If $I$ is the ideal of $F\{X\}[Y]$ generated by $\{x y \mid x \in X$ and $y \in Y\}$ and $R=F\{X\}[Y] / I$, then each $r \in R$ may be written as $r=a+f(X)+g(Y)$ for $a \in F, f(X) \in F\{X\}$, and $g(Y) \in F[Y]$, where $f(x)$ and $g(y)$ have no "constant" term. Clearly, $([R, R])=([(X),(X)]) \subseteq F\{X\}$ has cardinality $\alpha, Z(R)=F[Y]$ has cardinality $\beta$, as does $(Y) \subseteq Z$, and $[R:(Y)]=\alpha$. 
Note that $(Y)=\operatorname{ann}([R, R])$, ann $(Y)=(X), R /(Y) \cong F\{X\}$, and $R /(X) \cong F[Y]$, so as in Theorem 3, $R$ embeds in $F\{X\} \oplus F[Y]$. However, since $R$ contains no idempotents other than 0 and $1, R$ itself cannot be a direct sum of ideals.

We turn now to the notion of higher commutator and consider the same results as those above for higher commutators replacing $[R, R]$. It is convenient to discuss higher commutators in the more general context of Lie ideals which we define next.

Definition: A subgroup $(T,+)$ of $(R,+)$ is a lie ideal if $[T, R] \subseteq T$.

We note that if $A$ and $B$ are both Lie ideals then $[A, B]$ is also a Lie ideal because of the identity $[[x, y], r]=[[x, r], y]+[x,[y, r]]$. Consequently, expressions like $R,[R, R],[[R, R], R]$, or $[[R, R],[R, R]]$ are Lie ideals. One question we shall investigate is the cardinality of the ideal generated by these kinds of Lie ideals compared to the cardinality of the Lie ideal itself.

Definition: Given a ring $A$, a higher commutator $T$ of weight $k \geqslant 2$ is: $[A, A]$ if $k=2$; and for $k>2, T=[U, R]$ for $U$ a higher commutator of weight $k-1$, or $T=[U, V]$ for $U$ and $V$ higher commutators of weights $2 \leqslant m<k-1$ and $k-m$, respectively. The special higher commutators $A_{(i)}$ of weight $i$ are defined inductively by $A_{(2)}=[A, A]$ and $A_{(i+1)}=\left[A_{(i)}, A\right]$, and the higher commutators $A^{(i)}$ of weight $2^{i}$ are given by $A^{(1)}=[A, A]$, and $A^{(i+1)}=\left[A^{(i)}, A^{(i)}\right]$.

Note that any higher commutator of $A$ is a Lie ideal of $A$. The only higher commutator of weight 2 is $[A, A]=A^{(1)}=A_{(2)}$, and the only higher commutator of weight 3 is $A_{(3)}=[[A, A], A]$. There are two higher commutators of weight 4: $A_{(4)}$ and $A^{(2)}$, and because $[a, b]=-[b, a]$, three of weight $5: A_{(5)},\left[A^{(2)}, A\right]$, and $\left[A_{(3)}, A_{(2)}\right]$. By using the Jacobi identity $[[a, b], c]=[[a, c], b]+[a,[b, c]]$, it follows that $\left[A^{(2)}, A\right] \subseteq\left[A_{(3)}, A_{(2)}\right]$, and by induction on weight, that any higher commutator of weight $w$ is contained in $R_{(W)}$. Clearly, for each higher commutator $T$ of weight $k$ there is a multilinear and homogeneous polynomial of degree $k, f_{T}\left(x_{1}, \ldots, x_{k}\right) \in \mathbf{Z}\left\{x_{1}, \ldots, x_{k}\right\}$ with integer coefficients, so that $T$ is the additive subgroup generated by $\left\{f_{T}\left(a_{1}, \ldots, a_{k}\right) \mid a_{i} \in A\right\}$. For brevity we shall write $T=T(A)=f_{T}(A)$. Specifically, if $T=A^{(2)}, f_{T}\left(x_{1}, \ldots, x_{4}\right)=\left[\left[x_{1}, x_{2}\right],\left[x_{3}, x_{4}\right]\right]$ and when $T=\left[A_{(3)}, A_{(3)}\right], f_{T}\left(x_{1}, \ldots, x_{6}\right)=\left[\left[\left[x_{1}, x_{2}\right], x_{3}\right],\left[\left[x_{4}, x_{5}\right], x_{6}\right]\right]$.

We discuss a few examples which illustrate some basic differences between $R^{(i)}$ and $R_{(i)}$ and which will be useful for later reference. Recall from Example 3 that $M_{0}(F)$ is the ring of those countably infinite by countably infinite matrices over the field $F$ which have only finitely many nonzero entries. Writing $M_{0}(F)=\sum F e_{i j}$ and using $e_{i i}-e_{j j}=\left[e_{i j}, e_{j i}\right]$ and $e_{i j}=\left[e_{i k}, e_{k j}\right]$ when $i, j$ and $k$ are distinct, it is easy to see that for any higher commutator $T$ of weight $k>1, T\left(M_{0}(F)\right)=M_{0}(F)^{(1)}=$ $\sum_{i \neq j} F e_{i j}+\sum F\left(e_{i i}-e_{j j}\right)$. The same computation in $M_{n}(F)$ gives the same result unless 
$n=2=\operatorname{char} F$, in which case $M_{2}(F)_{(j)}=M_{2}(F)_{(2)}=$ \{matrices of trace zero\}, $M_{2}(F)^{(2)}=F I_{2}$, and $M_{2}(F)^{(j)}=0$ if $j>2$. If $R$ is the ring of upper triangular matrices in $M_{0}(F)$, then $[R, R]=\sum_{i<j} F e_{i j},[T, R]=T$ for any higher commutator $T$ of weight $w \geqslant 2$, and $R^{(k)}=\sum\left\{F e_{i j} \mid j \geqslant i+2^{k-1}\right\}$ when $k>1$. Similarly, if $A$ is the subring of upper triangular matrices in $M_{n}(F)$, then the $A^{(i)}$ strictly decrease until $A^{(k)}=0$ for suitably large $k$. Finally, if $B$ is the subring of all strictly upper triangular matrices in $M_{0}(F)$, then for any $T$ of weight $k, T(B)=\sum\left\{F e_{i j} \mid j \geqslant i+k\right\}$. For convenience, we state these observations for matrices as a lemma for future reference.

LEMma 2. Let $R=M_{n}(F)$ for $F$ a field and $n>1$. If $n=2=\operatorname{char} F$ then $R^{(2)}=F$ and $R^{(3)}=0$. If $n>2$ or $\operatorname{char} R \neq 2$, then $V(R)=R^{(1)}$ for any higher commutator $V$.

We investigate how the cardinality of a higher commutator $V$ is related to $[R: Z]$, as we did for $[R, R]$ in Theorem 3 , and also whether $\operatorname{card} V=\operatorname{card}(V)$. The latter question is certainly true by Theorem 1 for those higher commutators of the form $[T, R]$, but others constitute a considerable problem. Indeed, for arbitrary higher commutators $V$, we know of no counterexample to card $V=\operatorname{card}(V)$, but cannot prove this except for certain special cases, principally in prime $P I$ rings or for finite higher commutators in semiprime rings. As for the relation between card $V$ and $[R: Z]$, Example 1 shows that we must assume that $R$ is a semiprime ring and Example 3 shows that the bound $[R: Z] \leqslant 2^{\operatorname{card} V}$ is the best possible when $V$ is infinite. However, unlike Theorem 3 for $[R, R]$, when $V$ is a general higher commutator, there are difficulties relating $[R: Z]$ to card $V$. As we have observed just above, when $F$ is an infinite field with $\operatorname{char} F=2$, then $\left[M_{2}(F): F\right]=\operatorname{card} F$, but $M_{2}(F)^{(2)}=F I_{2}=Z\left(M_{2}(F)\right)$, and so any higher commutator of $M_{2}(F)$ of the form $[U, W]$ will be zero if either $M_{2}(F)^{(2)} \subseteq U$ or $M_{2}(F)^{(2)} \subseteq W$. Consequently, for higher commutators we must deal with the complication introduced by the presence of 2-torsion. Our next result relates the possibility that a higher commutator of $R$ is zero to the commutativity of $R$ and the presence of 2-torsion. We recall that the standard polynomial of degree $n$ is defined as $S_{n}\left(x_{1}, \ldots, x_{n}\right)=\sum_{\sigma}(-1)^{\sigma} x_{\sigma(1)} \ldots x_{\sigma(n)}$ where $\sigma$ runs over the symmetric group on $n$ letters. A ring satisfies $S_{n}$ if $S_{n}\left(r_{1}, \ldots, r_{n}\right)=0$ for all $r_{i} \in R$, and when $R$ is a semiprime ring it is well known that $R$ satisfies $S_{n}$ exactly when $R$ embeds in $M_{d}(C)$ for $C$ a commutative semiprime ring, with $d \leqslant n / 2[1$, Theorem 6.3.2, p.159] and [5, Theorem 2, p.35]). Finally, note that $S_{n}\left(r_{1}, \ldots, r_{n}\right)=0$ if some $r_{i}=r_{j}$ for $i \neq j$.

THEOREM 4. Let $R$ be a semiprime ring, and $T$ a higher commutator which is finite. Then $R$ contains only finitely many prime ideals $P_{1}, \ldots, P_{k}$ of finite index in $R$ so that $T\left(R / P_{i}\right) \neq 0$, and if char $R / P_{i} \neq 2$ or $R / P_{i}$ does not satisfy $S_{4}$, 
then for all higher commutators $V, V\left(R / P_{i}\right)=\left(R / P_{i}\right)^{(1)} \neq 0$. For any other prime ideal $P$ of $R, T(R / P)=0$ and either $R / P$ is commutative or $2 R \subseteq P, R / P$ satisfies $S_{4},(R / P)^{(2)} \subseteq Z(R / P)$, and $T \subseteq\left[R^{(2)}, R\right]$. In particular, $R$ is a subdirect sum of the semiprime images $R_{1}, R_{2}$, and $R_{3}$ so that: $R_{1}$ is finite, $T \cong T\left(R_{1}\right) ; R_{2}$ is commutative; and $2 R_{3}=0$, no prime image of $R_{3}$ is commutative, $R_{3}$ satisfies $S_{4}$, and $R_{3}^{(2)} \subseteq Z\left(R_{3}\right)$. Furthermore, $V\left(R_{1}\right)=R_{1}^{(1)}$ for any higher commutator $V$ when $R_{3} \neq 0$.

Proof: Let $T=T(R)=f_{T}(R)$ have weight $w$, assume that $P$ is a prime ideal of $R$ so that $T(R / P) \neq 0$ in $R / P$, and note that $T(R)+P=f_{T}(R)+P=$ $f_{T}(R / P)=T(R / P)$. To prove that $R / P$ is finite, it suffices to assume that $R$ is a prime ring, that $T(R) \neq 0$ is finite, and prove that $R$ is finite. If $\operatorname{card} T=k$, then $S_{k+1}\left(f_{T}\left(x_{11}, \ldots, x_{1 w}\right), \ldots, f_{T}\left(x_{k+11}, \ldots, x_{k+1} w\right)\right)$ is satisfied by $R$. A theorem of Rowen [5, Theorem 1, p.56] shows that $Z(R) \neq 0$, and the central localisation $S=R Z^{-1}$ of $R$ at $Z-\{0\}$ is a simple ring, finite dimensional over its centre $F=Q F(Z)[5$, Theorem 2, p.57]. But $T$ is a $Z$ module, $T$ is finite, and central elements in prime rings are regular, so it follows that $Z$ is finite. Hence $Z$ is a field and the finite dimensionality of $S=R Z^{-1}=R$ over $Z$ forces $R=M_{n}(Z)$ to be finite. When $2 R \neq 0$ or $R$ does not satisfy $S_{4}, V(R)=R^{(1)}$ for any higher commutator $V$, by Lemma 2 .

Returning to our semiprime ring $R$, suppose that $P_{1}, \ldots, P_{m}$ are distinct prime ideals, each satisfying $T\left(R / P_{i}\right) \neq 0$ in $R / P_{i}$. Since from above, each $R / P_{i}$ is a finite simple ring with identity, $R / \cap P_{i}$ is a finite semiprime ring with identity whose maximal ideals are exactly $\left\{P_{j} / \cap P_{i}\right\}$. Thus $R / \cap P_{i} \cong \bigoplus R / P_{i}$, and in particular, $m \leqslant$ $\operatorname{card} T\left(\bigoplus R / P_{i}\right)=\operatorname{card} T\left(R / \cap P_{i}\right)$. But $T\left(R / \cap P_{i}\right)=T(R)+\bigcap P_{i}$, so $m \leqslant \operatorname{card} T$. Consequently, there are only finitely many prime ideals $P_{1}, \ldots, P_{k}$ of $R$ satisfying $T\left(R / P_{i}\right) \neq 0$, and for each of them, $R / P_{i}$ is finite. Set $R_{1}=R / \cap P_{i}$.

Now let $P$ be a prime ideal of $R$ so that $T(R / P)=0$. To finish the proof it suffices to assume that $R$ is a prime ring with $T=0$, and show either that $R$ is commutative, or that $2 R=0, R$ satisfies $S_{4}, R^{(2)} \subseteq Z$, and $T \subseteq\left[R^{(2)}, R\right]$. The definition of $R^{(i)}$ shows that $R^{(j)} \subseteq R^{(i)}$ if $i \leqslant j$, so an easy induction proves that $R^{(m)} \subseteq T$ for some $m \geqslant 1$. Since $R$ satisfies the polynomial identity $f_{T}=0$, as above, $Z \neq 0$ and $S=R Z^{-1}$ is a simple ring, finite dimensional over its centre $F=Q F(Z)$. Now $[10$, Lemma 7 , p.120] proves that for Lie ideals $U$ and $W$ of $R$, if $[U, W] \subseteq Z$, then either $U \subseteq Z$ or $W \subseteq Z$, unless $2 R=0$ and $\operatorname{dim}_{F} S \leqslant 4$. Since all the $R^{(i)}$ are Lie ideals of $R$, we may apply this result to $0=R^{(m)}=\left[R^{(m-1)}, R^{(m-1)}\right]$ and use induction to show that either $R$ is commutative or it is not but $2 R=0$ and $\operatorname{dim}_{F} S=4$. In the latter case, either $S=M_{2}(F)$ or $S$ is a division algebra over $F$ and $S \otimes_{F} K=M_{2}(K)$ for $K$ a maximal subfield of $S$ [1, Corollary, p.96]. Thus $R$ embeds in $M_{2}(C)$ for $C$ a field, so it follows that $R$ satisfies $S_{4}$. Also, using Lemma $2, R^{(2)} \subseteq R \cap M_{2}(C)^{(2)} \subseteq R \cap C=$ 
$Z$. Furthermore $T\left(S \otimes_{F} K\right)=T(R) Z^{-1} \otimes_{F} K=0$, implying that $T \subseteq\left[R^{(2)}, R\right]$. Set $R_{2}=R / \cap P$ over all primes $P$ with $R / P$ commutative, and $R_{3}=R / \cap P$ over all primes $P$ with $T(R / P)=0$ but $R / P$ not commutative, and note that $R$ naturally embeds in $R_{1} \oplus R_{2} \oplus R_{3}$, so $T(R) \cong T\left(R_{1}\right)$. If $R_{3} \neq 0$, then $T \subseteq\left[R^{(2)}, R\right]$ so no simple summand of $R_{1}$ can be $M_{2}(F)$, and as we have seen, $V\left(R / P_{i}\right)=\left(R / P_{i}\right)^{(1)}$ for each such summand, and every higher commutator $V$. Hence $V\left(R_{1}\right)=R_{1}^{(1)}$ follows from $V\left(R_{1}\right)=V\left(R / P_{1}\right) \oplus \cdots \oplus V\left(R / P_{k}\right)$, and the proof of the theorem is complete.

Our first application of Theorem 4 shows that in a semiprime ring any finite higher commutator generates a finite ideal. Although we cannot prove this for arbitrary rings, we know of no counterexample.

Theorem 5. Let $R$ be a semiprime ring and $T$ a higher commutator of $R$.

(1) It $T$ is finite then the ideal $(T)$ is finite.

(2) If $T$ is finite and $R$ is 2-torsion free, then $R^{(1)}=V(R)$ for any higher commutator $V$.

(3) If $T$ is commutative then $2\left(R^{(1)}\right)=0$ and $R^{(2)} \subseteq Z$, the centre of $R$.

(4) If $T$ is commutative and $R$ is 2-torsion free, then $R$ is commutative.

Proof: Using Theorem 4, when $T$ is finite $R$ embeds in the direct sum of semiprime images $A \oplus B \oplus C$, with $A=R / P_{1} \oplus \cdots \oplus R / P_{k} \cong R / \bigcap P_{i}$ finite, $T\left(R / P_{i}\right) \neq$ $0, B$ commutative, $2 C=0$, and up to isomorphism $T=T(A)$. Consequently, $(T) \subseteq A$ is finite. If $R$ is also 2-torsion free, then $C=0$ and if $A \neq 0$ then by Lemma 2, each summand $R / P_{i}$ of $A$ satisfies $V\left(R / P_{i}\right)=\left(R / P_{i}\right)^{(1)}$ for any higher commutator $V$. Since $V(A)=V\left(R / P_{1}\right) \oplus \cdots \oplus V\left(R / P_{k}\right)$, it follows that $V(A)=A^{(1)}$, and so $V(R)=R^{(1)}$ since the commutativity of $B$ forces $V(B)=0$. When $T$ is commutative, apply Theorem 4 to $[T, T]=0$ to conclude that $A=0$, so $R^{(1)} \subseteq C$ and $R^{(2)} \subseteq C^{(2)} \cap R \subseteq Z(C) \cap R \subseteq Z(R)$. When $R$ is also 2-torsion free, $C=0$, so $R$ embeds in $B$ and is commutative.

By Theorem 5, when $T$ is finite and $R$ is 2-torsion free, any two higher commutators are equal to $R^{(1)}$. Without the restriction on torsion, for any two higher commutators, $V, W \subseteq\left[R^{(2)}, R\right], V=W$ since $V(C)=W(C)=0$ and $V(B)=W(B)=0$ force $V(R)=V(A)=W(A)=W(R)$. We turn now to the analogues of Theorem 1 and of Theorem 3 for higher commutators, but now need to assume that $R$ is 2-torsion free. After our proof, we give an example showing the need for the torsion assumption.

Theorem 6. Let $R$ be a 2-torsion free semiprime ring, $V$ an arbitrary higher commutator of $R$, and $T$ a higher commutator of $R$ with minimal cardinality $\alpha$. The following hold:

(1) There is an ideal $I$ of $R$ with $I \subseteq Z$, so that $[R: I] \leqslant 2^{\alpha}$ when $\alpha$ is infinite, and $[R: I]$ is finite when $\alpha$ is finite; 
(2) $\alpha \leqslant \operatorname{card} V \leqslant \operatorname{card}(V) \leqslant 2^{\alpha}$ when $\alpha$ is infinite;

(3) $V=T=R^{(1)}$ when $\alpha$ is finite; and

(4) $R$ is the subdirect sum of 2-torsion free semiprime images $R_{1}$ and $R_{2}$ with $R_{1}$ commutative and card $R_{2} \leqslant 2^{\alpha}$ when $\alpha$ is infinite, and when $\alpha$ is finite, $R_{2}$ is finite and $R \cong R_{1} \oplus R_{2}$.

Proof: Note that $\{\operatorname{card} V \mid V$ is a higher commutator of $R\}$ has a minimal element $\alpha[6$, Theorem 23, p.53]. If some $V=0, R$ is commutative by Theorem 5 and the theorem holds. If $\alpha>1$ is finite then (3) holds by Theorem 5 . Now assume that $\alpha$ is infinite and consider the higher commutator $U=[T, R] \subseteq T$. If $U=0$, then $R$ is commutative by Theorem 5 , forcing the contradiction $T=0$. Thus $U \neq 0$ and clearly $\operatorname{card} U \leqslant \alpha$, so Theorem 1 shows that for $W=(U)$, card $W \leqslant \alpha$. As in the proof of Theorem 2, $R$ acts by left multiplication on $W$ as elements of $\operatorname{End}(W,+)$, $I=l(W)$ satisfies $[R: I] \leqslant 2^{\alpha}$, and $I \cap W=0$ because $R$ is semiprime. Note that if $U=f_{U}(R)$, then $U(I)=f_{U}(I) \subseteq I \cap W=0$. Since $I$ is an ideal of $R$ it is either (0) or is a 2-torsion free semiprime ring, so applying Theorem 5 forces $I$ to be commutative. It follows easily [2, Corollary, p.7] that $I \subseteq Z$, proving (1) when $\alpha$ is infinite. Applying Corollary 1.2 gives card $([R, R]) \leqslant 2^{\alpha}$, and since $(V) \subseteq([R, R]), \operatorname{card}(V) \leqslant 2^{\alpha}$ follows and the minimality of $\alpha$ yields $\alpha \leqslant \operatorname{card} V \leqslant \operatorname{card}(V) \leqslant 2^{\alpha}$, proving (2).

Finally, with $I=l(W)$ as above, set $J=l(I)$ and note that as in the proof of Theorem 3,I and $J$ are semiprime ideals of $R, R$ is the subdirect sum of $R_{1}=R / J$ and $R_{2}=R / I$, and card $R_{2} \leqslant 2^{\alpha}$. Each $R_{i}$ is easily seen to be 2-torsion free, so $R_{1}$ is commutative by Theorem 5 because $U(R) \subseteq W \subseteq J$. When $\alpha$ is finite, $W$ is finite by Theorem 1, so a summand of $R$ and $R \cong I \oplus W$, making $W=J=R_{2}$ and $I=R_{1}$. $\square$

Recall $[6$, p.66] that the Generalised Continuum Hypothesis (GCH) says that for an infinite cardinal $\alpha$ there are no cardinals strictly between $\alpha$ and $2^{\alpha}$. Using this gives a sharper version of part of Theorem 6 .

Corollary 6.1. Let $R$ be a 2-torsion free semiprime ring with an infinite higher commutator. Assuming the GCH, for some infinite cardinal $\alpha$ and any higher commutator $V$, either card $V=\alpha$ or $\operatorname{card} V=2^{\alpha}$, and $\operatorname{card}(V)=\alpha$ or $\operatorname{card}(V)=2^{\alpha}$.

We record an easy example which shows that neither part (1) nor part (3) or Theorem 6 need hold in the presence of 2-torsion.

EXAMPLE 5. Let $R=M_{2}(F)$ for $F$ a field with $\operatorname{char} F=2$ and $\operatorname{card} F=\alpha$, infinite and not countable. Since $\operatorname{char} F=2$, we have seen that $R^{(3)}=0$, so is finite, but $[R: Z]=\alpha$ and $Z$ contains no nonzero ideal of $R$. Also, $R$ is a simple ring so cannot be a subdirect sum as in Theorem 3. Taking $S=R \oplus M_{n}(K)$ for $K$ a field with card $K \leqslant \beta<\alpha$ and either $n>2$ or $n=2$ and char $K>2$, gives a similar example but with card $S^{(3)}=\beta$, or finite and not zero. 
Theorem 6 tells us that in a 2-torsion free semiprime ring with an infinite higher commutator, there are only two possible cardinalities for these commutators, assuming the GCH. Of course for $R=M_{2}(F)$ with $F$ a finite field and char $F=2, R^{(1)} R^{(2)}$ and $R^{(3)}$, have different cardinalities. We present an example to show that different infinite cardinals can in fact occur.

Example 6. As in Example 2, let $A=\prod_{E} M_{2}(F)$, the infinite direct product of $\alpha$ copies of $M_{2}(F)$, where $F$ is a finite or countable field, $\alpha$ is any infinite cardinal, and card $E=\alpha$. If $D \subseteq A$ is the direct sum of $\alpha$ copies of $M_{2}(F)$, let $B=\{g \in A \mid g(i)$ is upper triangular for all $i \in E\}$, and set $R=D+B$. It is straightforward to check that: $R$ is a semiprime ring; $\operatorname{card} D=\alpha ; \operatorname{card} B=\operatorname{card} R=2^{\alpha} ; \operatorname{card} R^{(1)}=2^{\alpha}$, since it contains $B^{(1)}$, all functions $g: E \rightarrow F e_{12}$; and card $R^{(2)}=\alpha$, since $R^{(2)} \subseteq D$. Similarly, one can replace $M_{2}(F)$ with $M_{n}(F)$ for $n>2$, in which case for suitably large $s \geqslant 2, \operatorname{card} R^{(*)}=\alpha$ but $\operatorname{card} R^{(t)}=2^{\alpha}$ if $1 \leqslant t<s$.

The ring in Example 6 satisfies the standard identity $S_{4}$. We shall show that in a prime ring satisfying a standard identity, all infinite higher commutators have the same cardinality. We give an example of a prime ring in which there are higher commutators with different infinite cardinals.

EXAmple 7. We consider a modification of Example 3. Let $H$ be the ring of all row finite, countably infinite by countably infinite matrices over a countable field $F$. Denote by $M_{0}(F)$ the subring of $H$ of all matrices having only finitely many nonzero entries, and by $A$ the subring of $H$ of all block diagonal $2 \times 2$ upper triangular matrices. Observe that $M_{0}(F)$ is a countable ring, $A$ is uncountable, $A M_{0}(F)+M_{0}(F) A \subseteq M_{0}(F)$, and that $R=M_{0}(F)+A$ is a prime ring. Now $R^{(1)}$ is uncountable, but $R^{(2)} \subseteq M_{0}(F)$ is countable. A similar example can be constructed for any infinite cardinal $\alpha$ and $2^{\alpha}$ by using $\boldsymbol{\alpha} \times \boldsymbol{\alpha}$ matrices.

The dichotomy represented by Theorem 6 and the last two examples can fail for rings which are not semiprime. If $R$ is the ring of all (strictly) upper triangular matrices in $M_{n}(F)$ with $n$ large and $F$ a finite field, then $\left\{R^{(i)}\right\}$ (or $\left\{R_{(i)}\right\}$ ) strictly decreases in cardinality, until $i$ is sufficiently large. We show how to construct a nilpotent example with an arbitrary finite chain of infinite cardinals.

EXAMPLE 8. Let $\alpha_{1}>\alpha_{2}>\cdots>\alpha_{n}$ be infinite cardinals. For each $1 \leqslant i \leqslant n$ let $F_{i}=J\left\{X_{i}\right\}$ be the free algebra over the integers $J$ in a noncommutative set $X_{i}$ of indeterminates with card $X_{i}=\alpha_{i}$. Set $R(i)=\left(X_{i}\right) / H$, where $H$ is the ideal of $F_{i}$ generated by all monomials of degree $i+2$. Note that $R(i)$ is nilpotent of index $i+2$, and $\operatorname{card} R(i)=\operatorname{card} R(i)_{(j)}=\alpha_{i}$ for $j<i+2$. Therefore, if $R=R(1) \oplus R(2) \oplus \cdots \oplus R(n)$, then for $2 \leqslant i \leqslant n+1, R_{(i)}=R(i-1)_{(i)} \oplus \cdots \oplus R(n)_{(i)}$ and card $R_{(i)}=\alpha_{i-1}$.

The last results we obtain are conditions under which $\operatorname{card} T=\operatorname{card}(T)$ when $T$ 
is an infinite higher commutator. We state first an immediate consequence of Theorem 1 , using the fact that any higher commutator is a Lie ideal.

THEOREM 7. If $T$ is a higher commutator of $R$ having the form $T=[A, R]$ for $A$ a higher commutator, then $\operatorname{card} T=\operatorname{card}(T)$ when $T$ is infinite, and $\operatorname{card}(T)$ is finite when $T$ is finite.

In Theorem 5 we saw that when $R$ is a semiprime ring, any finite higher commutator generates a finite ideal. Although we cannot prove this in general, we can show in general that the subring generated is finite. Of course, the subring generated by an infinite set $A$ in a ring $R$ has the same cardinality as $A$.

THEOREM 8. If $T$ is a higher commutator in $R$ and is finite, then $\langle T\rangle$, the subring $T$ generates, is also finite.

Proof: By Theorem $7, I=([T, R])$ is finite, since $[T, R] \subseteq T$ is finite. In $R / I$, $T(R / I)=T(R)+I$ is central from the definition of $I$. Now $T(R)=f_{T}(R)$ is torsion, as in Lemma 1, since $n f_{T}\left(r_{1}, \ldots, r_{k}\right)=f_{T}\left(n r_{1}, \ldots, r_{k}\right)$ and $T$ is finite. Consequently, $T(R / I)$ consists of finitely many central torsion elements, so $\langle T(R / I)\rangle$ is finite. Thus in $R,\langle T\rangle+I$ is finite, so $\langle T\rangle$ is finite.

We end the paper with two related special cases.

Theorem 9. Let $R$ be a simple ring and $T$ a higher commutator of $R$. Then either:

(1) $R$ is finite;

(2) $\operatorname{card} T=\operatorname{card}(T)=\operatorname{card} R$; or

(3) $T=0$ and either $R$ is a field or $\operatorname{dim}_{Z} R=4,2 R=0$ and $R^{(2)}=Z$.

Proof: Assume throughout that $R$ is infinite. If $[T, R] \neq 0$, then by Theorem $7, \operatorname{card}([T, R])=\operatorname{card}[T, R] \leqslant \operatorname{card} T$, so (2) holds since $R$ is simple and $R=([T, R]) \subseteq(T)$. Suppose then that $[T, R]=0$ and apply Theorem 4 to the higher commutator $[T, R]$ to conclude that either $R$ is commutative, or that $2 R=0, R$ satisfies $S_{4}$, and $R^{(2)} \subseteq Z$. When $R$ is commutative it is a field and $T=0$. Finally, when $2 R=0$ and $R$ satisfies $S_{4}$, but is not commutative, a theorem of Kaplansky [5, Theorem p.17] yields $\operatorname{dim}_{Z} R=4$, and also $R^{(2)}=Z$ since $R^{(2)}$ is a $Z$ module and $Z$ is a field. Therefore, when $T=0,(3)$ holds, and if $T \neq 0$, then $\operatorname{card} T=\operatorname{card} Z=\operatorname{card} R$, since $T$ is a $Z$ module and $R$ is infinite, so (2) holds.

Our last result is for prime rings satisfying a polynomial identity. If one is unfamiliar with this notion, then one may assume instead that the ring satisfies a standard identity $S_{n}$ as defined above, an equivalent statement for prime rings. Recall that by Example 3, for a prime ring not satisfying a polynomial identity, $\operatorname{card} T<\operatorname{card} R$ can occur. Our result shows that in a prime ring satisfying a polynomial identity, all infinite 
higher commutators have the same cardinality as the ring itself.

THEOREM 10. Let $R$ be a prime ring satisfying a polynomial identity. If $T$ is a higher commutator of $R$, then either:

(1) $R$ is finite;

(2) $\operatorname{card} T=\operatorname{card}(T)=\operatorname{card} R$; or

(3) $T=0$ and either $R$ is commutative or $2 R=0, R$ satisfies $S_{4}$, and $R^{(2)} \subseteq Z$.

Proof: As we have seen in the proof of Theorem 4, since $R$ satisfies a polynomial identity, we may conclude that $Z \neq 0$ and that the central localisation $S=R Z^{-1}$ of $R$ at $Z-\{0\}$ is a simple ring, finite dimensional over its centre $Q F(Z)$. Note that if $T=$ $f_{T}(R)$, then $T Z^{-1}=f_{T}(R) Z^{-1}=f_{T}(S)=T(S)$. The result now follows by applying Theorem 9 to $S$ and $T(S)$. Specifically, if $Z$ is a field, then $R=S$ and $T=T(S)$. If $Z$ is not a field then $\operatorname{card} S=\operatorname{card} R$ and $\operatorname{card} T(S)=\operatorname{card} T(R)$, so if (2) of Theorem 9 holds for $S$ and $T(S)$, then $\operatorname{card} R=\operatorname{card} T=\operatorname{card}(T)$. Finally, if $T(S)=0$, then $T=0$, so if $S$ is a field $R$ is commutative, and if $2 S=0$ and $\operatorname{dim}_{Q F(Z)} S=4$, then $S$, and so $R$, satisfies $S_{4}$, and $R^{(2)} \subseteq S^{(2)} \cap R=Q F(Z) \cap R=Z$.

We observe that in part (3) of Theorem 10, one cannot conclude that $R^{(2)}=Z$ as in Theorem 9. For example, if $C$ is a commutative domain with a proper nonzero ideal $H$ and if char $C=2$, let $R=\left\{A \in M_{2}(C) \mid A_{12} \in H\right\}$. Then $R$ is a prime ring satisfying the standard identity $S_{4}$ and $R^{(2)}=H I_{2} \neq C I_{2}=Z(R)$.

Finally, in closing we note that in trying to prove that $\operatorname{card} T=\operatorname{card}(T)$ for any infinite higher commutator in an arbitrary ring, one may assume that $T \subseteq Z$. By Theorem 1, we know that $\operatorname{card}([T, R])=\operatorname{card}[T, R]$, or both are finite, so if $R^{\prime}=$ $R /([T, R])$ it suffices to prove that $\operatorname{card} T\left(R^{\prime}\right)=\operatorname{card}\left(T\left(R^{\prime}\right)\right)$. Unfortunately, we cannot make use of this additional assumption.

\section{REFERENCES}

[1] I.N. Herstein, Noncommutative rings, Carus Mathematical Monographs 15 (Mathematical Association of America, 1968).

[2] I.N. Herstein, Rings with involution (University of Chicago Press, Chicago, 1976).

[3] Y. Hirano, 'Some finiteness conditions for rings', Chinese J. Math. 16 (1988), 55-59.

[4] Y. Hirano, 'On a problem of Szász', Bull. Austral. Math. Soc. 40 (1989), 363-364.

[5] N. Jacobson, PI-algebras, Lecture Notes in Mathematics 441 (Springer-Verlag, Berlin, Heidelberg, New York, 1975).

[6] I. Kaplansky, Set theory and metric spaces (Chelsea Publishing Company, New York, 1977).

[7] K. Koh, 'On properties of rings with a finite number of zero divisors', Math. Ann. 171 (1976), 79-80. 
[8] C. Lanski, 'Rings with few nilpotents', Houston J. Math. 18 (1992), 577-590.

[9] C. Lanski, 'On the cardinality of rings with special subsets which are finite', Houston J. Math. 19 (1993), 357-373.

[10] C. Lanski and S. Montgometry, 'Lie structure of prime rings of characteristic 2', Pacific J. Math. 42 (1972), 117-136.

[11] J. Lewin, 'Subrings of finite index in finitely generated rings', J. Algebra 5 (1967), 84-88.

\footnotetext{
Department of Mathematics

University of Southern California

Los Angeles, CA 90089-1113

United States of America

e-mail: clanski@ mtha.usc.edu
} 